Este é um artigo publicado em acesso aberto sob uma licença Creative Commons

https://creativecommons.org/licenses/by-nc/4.0/

\title{
Puntuando algunos de los desafíos de las universidades latinoamericanas en el futuro presente ${ }^{1}$
}

\author{
Aura González Serna ${ }^{1}$ \\ Edvânia Torres Aguiar Gomes ${ }^{2}$ \\ Dweison Nunes Souza Silva ${ }^{3}$
}

Resumen: Este artículo busca reflexionar sobre los desafíos y caminos de la educación superior, en especial, el papel actual y futuro de las universidades latinoamericanas en el proceso de formación de individuos y sociedades. Para tanto, recoge a las consideraciones debatidas en el panel: los desafíos de la universidad y la educación en América Latina hoy, en la Universidad Autónoma Latinoamericana Unaula - Colombia en 2014, así como nexo con autores y publicaciones oficiales sobre el tema. La universidad en la condición de institución social y destinada a metabolizar procesos de educación democrática, necesita abandonar ciertos conceptos intencionalmente construidos a lo largo de la historia y acercarse con urgencia a la sociedad. Su desafío está en la necesidad de romper con los paradigmas del conocimiento estructuralista, que en la contemporaneidad homogeniza la forma como se construyen individuos y sociedad, aspecto vinculado en grande medida a la noción de sociedad del conocimiento, utilizándose de las llamadas innovaciones tecnológicas. Finalmente, el éxito de ese enfrentamiento depende de los vínculos e imperativos que presente y futuro están siendo creados con la sociedad en general, puntos clave por los cuales la educación superior necesita responder a las necesidades de todos los sectores da sociedad. En este sentido, la universidad, ejerce un papel fundamental para la objetivación de la internacionalización de la educación superior, en la medida en que permite a los estudiantes vivenciar, de manera profunda y transformadora, los intercambios a través de sus programas de cooperación internacional en niveles de pre-grado, maestría y doctorado, punto esencial para la formación de individuos críticos y más preparados a las demandas presente futura en sus diferentes escalas.

Palavras-chave: Educación superior. Cooperación internacional. Universidad.

\section{Scoring some of the challenges of latin american universities in the present future}

Abstract: This article seeks to reflect on the challenges and paths of higher education, especially the current and future role of Latin American universities in the process of training individuals and societies. In order to do so, it mainly includes considerations debated in the panel: the challenges of university and education in Latin America today, carried out at the Universidad Autónoma Latinoamericana - Unaula - Colombia in 2014, as well as a link with authors and official publications on the subject. The university in the condition of social institution and destined to metabolize processes of democratic education, needs to abandon certain concepts intentionally constructed throughout the history and approach with urgency to the society. Its challenge lies in the need to break with the paradigms of structuralist knowledge, which contemporaneously homogenizes the way individuals and society are constructed, an aspect closely linked to the notion of a knowledge society, using the so-called

${ }^{1}$ Este artículo hace parte de investigaciones resultantes de la cooperación internacional entre la Universidade Federal de Pernambuco (UFPE), Brasil y Universidad Pontificia Bolivariana (UPB), Colombia, específicamente, por medio de los programas de pos grado en Desarrollo y Medio Ambiente - PRODEMA UFPE y Maestría en Desarrollo - UPB, bien como de los Grupos de investigación Sociedade e Natureza Nexus - UFPE y Territorio - UPB. 
technological innovations. Finally, the success of this confrontation depends on the links and imperatives that present and future are being created with society in general, key points for which higher education needs to respond to the needs of all sectors of society. In this sense, the university plays a fundamental role in objectifying the internationalization of higher education, insofar as it allows students to experience, in a profound and transformative way, exchanges through their international cooperation programs in levels of pre-degree, master's and doctorate, essential point for the formation of critical individuals and more prepared to the future present demands in its different scales.

Key words: Higher education. International cooperation. University.

\section{Pontuando alguns dos desafios das universidades latino-americanas no presente futuro}

Resumo: Este artigo busca refletir sobre os desafios e caminhos da educação superior, especialmente, o papel atual e futuro das universidades latino-americanas na formação de indivíduos e sociedades. Para tanto, recorre as considerações debatidas no painel: los desafíos de la universidad y la educación en América Latina hoy, en la Universidad Autónoma Latinoamericana - Unaula - Colombia, em 2014, bem como a autores e publicações sobre o tema. A universidade, na condição de instituição social projetada para metabolizar processos de educação democrática, precisa abandonar certos conceitos construídos intencionalmente ao longo da história e aproximar-se com urgência da sociedade. Seu desafio reside na necessidade de romper com os paradigmas do conhecimento estruturalista que na contemporaneidade homogeneíza a forma como os indivíduos e a sociedade são construídas, aspecto em grande medida relacionado com a noção de sociedade do conhecimento sob a égide das chamadas inovações tecnológicas. Finalmente, o êxito a esse enfrentamento depende dos vínculos e imperativos que, tanto no presente quanto no futuro, estão e venham a ser criados com a sociedade. Aspectos centrais pelos quais a educação superior precisa responder às necessidades de todos os setores da sociedade. Neste sentido, a universidade desempenha um papel fundamental na objetivação da internacionalização do ensino superior, na medida em que possibilita os estudantes vivenciar experiências intensas e transformadoras, por meio de seus programas de cooperação internacional em níveis de graduação, mestrado e doutorado, essenciais para a formação de indivíduos críticos e mais preparados para responder às exigências presente e futuras em diferentes escalas.

Palavras-chave: Educação superior. Cooperação internacional. Universidade. 


\section{Introducción}

En 2014, a partir de la invitación de La Universidad Autónoma LatinoamericanaUnaula, se debatió sobre los desafíos de la formación superior en el complejo mundo que hemos construido actualmente ${ }^{2}$. Mundo que está, más que en ningún otro tiempo histórico, compenetrado y en efervescencia como manifestación de reivindicaciones sociales. Viene al caso la lectura de un editorial de Ignacio Ramonet, publicado en la versión divulgada en Colombia de Le Monde Diplomatique en mayo de 2013 y titulado "El mundo en 2030". Rutinariamente, según Ramonet, al realizarse el cambio de mandato presidencial en Estados Unidos, el nuevo presidente (para el caso el 21 de enero/2013) encuentra en su escritorio un informe de inteligencia que tiene la intencionalidad de orientar la ruta, en horizonte prospectivo, de la visión desde una central de inteligencia, sobre los dilemas que deberá considerar la misión que continuará la defensa de los intereses de Estados Unidos en el escenario mundial.

Según Ramonet (2013), todas las cancillerías del mundo conocen ese informe y es compartido por las agencias de inteligencia de EEUU y para su construcción son consultados muchos países y especialistas de varias universidades, con énfasis en escala global. Es decir, que no es una lectura sólo de Occidente, la que sirve de materia prima para la elaboración del mismo. Según la fuente referenciada la principal constatación es el declive en la hegemonía de Occidente. Por vez primera desde el siglo XV, los países occidentales están perdiendo poderío frente a potencias emergentes. Estados Unidos deberá dividir su poderío con China y ya no podrá sostener hegemonía militar a semejanza del fin de la llamada Guerra Fría. Vamos hacia un mundo multipolar, habrá reacomodo en las disputas y países emergentes estarán realineándose. La profunda crisis económico-financiera contemporánea recreará polos hegemónicos regionales, con tendencia a transformarse en grupos de influencia mundial.

Este parafraseado del informe que inspira el editorial de Le Monde, nos ratifica en el argumento que niega la espontaneidad, la auto-actividad y, en ese mismo sentido, la utilización del término "por naturaleza" para referirnos a las concepciones de mundo en cada

\footnotetext{
${ }^{2}$ Este artículo recoge, de entre otros, a las reflexiones presentadas en el panel: Los desafíos de la universidad y la educación en América Latina hoy, en el marco del: Encuentro Pensamiento latinoamericano: un desafío al despliegue de lo posible, Homenaje al maestro Hugo Zemelman Merino, realizado en Medellín los días 12 y 13 de mayo de 2014 en la Universidad Autónoma Latinoamericana en Medellín.
} 
coyuntura histórica y al cambio social que visibilizan los conflictos, suscitados por relaciones de fuerzas en permanente contradicción y antagonismos, a partir de los intereses en disputa.

Raúl Zibechi (2015) explicaría estas propiedades exacerbadas del ser social a través de lo que denomina potencia-latencia que anida medularmente en el mundo de los que se sienten desposeídos, discriminados, aplazados. Resulta en dinámicas que arraigan luchas y que recrean constantemente expresiones, tácticas de sobrevivencia y acumulan conocimientos en contravía a las concepciones por reproducir hegemonías que sostengan el statu quo. La perspectiva de la geo-política para interpretar el mundo en el que vivimos, parece referirse a lo que se impone, a lo que se implanta, a lo que no tiene marcha atrás. Los movimientos de sociedad no serían instigados por conquistas, por poderes populares que incomodan las temporalidades del sistema que se haya dominante. Un mundo beligerante está en pleno desencantamiento. Es decir, la historia no es lineal, no está escrita y la geo-política también tiene su etimología como disciplina lanzada con intencionalidad y perspectiva, en la representación del mundo. Las interpretaciones desde la geo-política no alcanzan a compenetrar los intersticios del mundo que se re-crea complejo desde la totalidad que contiene los poderes populares.

En ese sentido, los políticos, los economistas no consiguen anticipar los grandes acontecimientos de los últimos 30 años. La teoría económica ha sido puesta a prueba. Algunos mandatarios y líderes empresariales tal vez han tenido que consultar a astrólogos antes de decidir dónde invertir y cuáles alianzas profundizar. Nada más representativo para evidenciar la confusión de la denominada grande política, que la amenaza para el conjunto de Europa sobre la homeostasis de la Unión Europea, dados los complejos antagonismos en los proyectos de sociedad que pretendan un ethos de sociedad único frente a los dilemas del mundo contemporáneo (UNESCO, 2015).

Retomando el editorial de Ramonet (2013) en Le Monde, éste se refiere también a dinámicas por la universalización de tecnologías, técnicas, conocimientos y al desbordante poder de visibilizar en tiempo real las luchas populares y la penetración por las redes a las captaciones clandestinas de informaciones de origen electromagnético. Manifestaciones referidas a conflictos hídricos, la artificialización de la naturaleza, la explotación del petróleo y del gas de esquisto, a partir de la implementación de técnicas de fracturación hidráulica, a niveles nunca imaginados y en clara demanda por homogeneizarse en territorios, globalmente.

En ese contexto de imposibilidad para predecir futuros, cuando no son los individuos aisladamente quienes construyen el mundo, sino los seres en sociedad los que saturan de intencionalidades y causalidades, deparados con sus posibilidades, la incesante búsqueda por 
satisfacer necesidades que se han tornado complejas, a través de explotación de recursos, de aprovechamiento de conocimientos y la acumulación de técnicas-tecnologías para hacer uso de artefactos cada vez más sofisticados en cuanto a las mediaciones que representan...¿cuál es el lugar del conocimiento en lo contemporáneo?, ¿qué rol se espera de las universidades? La expectativa que Occidente configuró de las universidades, siendo mediación para la transformación del mundo, ¿está en crisis?, ¿será, en el 2030, de actualidad la universidad en la disputa entre debates, dentro del amplio espectro de ofertas de especializaciones, posgrados, formación superior?, ¿será que en las universidades se estarán construyendo problematizaciones que permitan representar los movimientos de la sociedad no solo en perspectiva geo-política, sino en la potencia-latencia de las innumerables resistencias de los que recrean tácticas cotidianas para reproducir sus existencias?, ¿serán las universidades invitadas de piedra por los comandos de diversos capitales para legitimar pseudoconocimientos a modo de conocimientos?, ¿a qué intereses la ignorancia sirve?, ¿cuáles actitudes y prácticas contribuyen a la preservación de la vida en el planeta y a cuáles proyectos de existencia protege lo que las universidades reproducen y legitiman?

En realidad todas esas preguntas no pretenden ser respondidas en este artículo, ni hay salida premonitoria que pueda ser promesa de reposo. De las universidades debe revelarse un oráculo para poner en las manos de los instrumentalizadores, las estrategias para representar salvamentos ante las penurias de la vida cotidiana. Vaya trama y trampa en este círculo vicioso, por no decir trágico que viene representando un horizonte ilusorio.

Argumentos, por ejemplo como la competitividad justa, acumulación de capitales a través de los gastos sociales inspirados en políticas sociales de focalización en las camadas más vulnerables de la sociedad, reproducen contenidos de formación profesional que esencializan modos de instrumentalizar practicas dirigidas a la utilización de recursos públicos en perspectiva del beneficio de las privatizaciones y, por ende, en la profundización de las desigualdades sociales. El garante de lo público pasa a ser el mercado financiero y de capitales a través de bancos y fondos estatales. Por la vía de la deuda pública se sustenta la valorización del capital. Las voces se hacen activas sobre temas de lucros y el interés mediático parece reproducir la moda de demandarle a las universidades que vinculen sus contenidos a las respuestas que el medio técnico reclama para entrar a operar con realidades que buscan más de lo mismo, es decir acumulación de capitales.

Fattorelli (2015) afirma y explica que habiendo participado en auditorias sobre deuda pública de países como Ecuador, Grecia, Argentina, Brasil, los denominados “métodos” para favorecer el sistema privado, no difieren. Entonces, será posible que el socio-metabolismo que 
recrea las ofertas desde las universidades pueda decantar en obviedades tales como: hagan lo que hagan estará mal, sin embargo, como hay que continuar la misión institucional, más le vale a las universidades formar dentro de los cánones y modelos esencializados. El mundo va hacia el colapso y no hay valor-poder humano que pueda impedirlo. El dinero público privatizado en escala global no resolverá nada. ¿Será esa la predicción que podremos realizar?

Marx (1867) en el siglo XIX ${ }^{3}$ enfatizaba sus deconstrucciones sobre el mundo en el que le tocó vivir ${ }^{4}$, demostrando que el modo de formación social capitalista era estructuralmente inestable. Su motor de transmisión estaba dirigido a reproducir cada vez grandes auges y recesiones, diferenciándose en la temporalidad de estos ciclos y en el horizonte, anticipando la destrucción del mismo, solo que en circunstancias y tiempos históricos no predecibles, de acuerdo a los movimientos del sistema mundo (WALLERSTEIN, 2015) del capitalismo.

De ese modo, Marx estaba obsesionado por evidenciar lo que las manifestaciones del capitalismo en cadena y en escala podrían anunciar de su naturaleza anárquica, caótica y de barbarie frente a la promesa incumplida de la humanización de la vida. Esto porque, él advertía, que el capitalismo destruye su propia base social, a través de la precarización devastadora que propaga. El sentimiento de que las vidas en el planeta están sometidas a controles que escapan a nuestras fuerzas, amplía miedo, incertidumbre y desesperanza. La moralidad como línea base de modelos sobre familia, instituciones, relaciones laborales, sociabilidad, viene siendo desenmascarada. La epidemia se instaló a partir del proyecto individualista y consumista que inspiró las tesis de la sociedad capitalista. Todas las generaciones enfrentadas a una vida de profunda zozobra.

¿Cómo rescatar la perspectiva de la ética que es antagónica al tamiz de fondo argumentado por la moralidad, desde donde se han pretendido abrazar valores? La ética tiene su foco medular en el nosotros, en el somos, en todo el mundo, en la comprensión ontológica del tiempo histórico y no del tiempo de la vida de cada individuo. Es decir, todos somos

\footnotetext{
${ }^{3}$ Marx en el Capitulo 23 del Tomo I de El Capital denominado "La Ley general de la acumulación capitalista" explica el proceso que lleva a la disminución relativa del capital variable respecto al capital constante, vinculándolo con la tendencia a las crisis recurrentes del capitalismo. Para abordar las crisis, se explica detalladamente en la sección tercera del Tomo III (Cap. 13 al 15).

${ }^{4}$ Es en la primera mitad del S. XIX que aparecen los pre-requisitos generales a partir de los cuales se articulan las grandes matrices económicas, culturales y sociales del mundo contemporáneo. Entre la preparación ideológica de la Revolución Francesa y las sublevaciones de trabajadores de 1848, emergieron los núcleos básicos de aquello que identificamos como razón moderna, con su pesada carga de paradojas y contradicciones. La sociedad burguesa instaura profundas alteraciones en las relaciones sociedad-naturaleza, lo que profundizó en la denominada Revolución Industrial.
} 
mundo, el mundo somos todos, lo que pase con el mundo me involucra, antes que individuos en la naturaleza de la sociedad, somos seres sociales compenetrados en relaciones inseparables con todos los seres que habitan el planeta, Freire (1993). Las dicotomías en esas concepciones, las jerarquizaciones en esas aprehensiones, el entendimiento de la vida como sucesión de etapas en la que cada una es un paso adelante respecto a la anterior, sólo han servido para devastar la vida generosa en posibilidades en el planeta.

Tanto las teorías que irrumpen desde concepciones de autoritarismo, de populismo, de consensuar en la marginalidad, del cenit anhelado del socialismo de la acumulación, del desarrollo sostenible, constituyen tramas que representan más de lo mismo, frente a la urgente necesidad de recomposición de un movimiento practico-teórico-práctico en la producción de pensamiento de rigor centrado en la ontología del ser social histórico, con sus objetivaciones, subjetividades y en profunda relación de inter-dependencia con la naturaleza y sus entornos. Los meta-relatos, la meta-política, la pequeña política que define componendas acaparando usufructos que individualizan la riqueza social, sirven para instrumentalizar funcionalidades, disfrazando la misión de la universidad en ese futuro ausente que configura.

Recordando las intervenciones del panel en Unaula, a partir del audio grabado, se plantean dos planos, y en el marco de esos planos construye el siguiente enunciado que antecede a su pregunta:

En un plano A, Occidente y la universidad como una institución que efectivamente ha generado todas las tecnologías del control que le permiten asumirse como la gran institución que incluso se separó de la modernidad y se autodeterminó como la gran institución regularizadora sobre todo el globo y en particular en América Latina, es decir, es la universidad la que controla todas las estrategias de aprendizaje en América Latina, y en esa medida ha institucionalizado los métodos de Occidente como la única forma de planteamiento de un conocimiento occidentalizado.

Y en un plano B, lo que en tiempos recientes ustedes han denominado "modernidad" y "colonialidad"; cuando digo ustedes me refiero a los autores que recientemente han puesto en la otra escena, en la otra orilla, la cosmovisión según la cual existen pensamientos alternativos, no pensamientos únicos, y entonces han dicho que no son una nueva hegemonía, sino que son una red de modernidad y de colonialidad, y allí han descollado autores importantes, el profesor Arturo Escobar, Ramón Grosfoguel, autores que de alguna manera han sido abordados por gusto o por debate.

Pues bien, en el plano A, Occidente, la universidad ha occidentalizado el pensamiento único, ha indicado que la universidad tiene protocolos, tiene estándares, tiene modelos, y que 
en esa medida los currículos son la extensión de la universidad de quienes vamos a beber en calidad de sujetos del Occidente, bien porque somos profesores que ejercemos controles y a su vez la universidad nos controla, bien porque somos estudiantes que hacemos uso de las metodologías de la universidad, bebemos del currículo, obtenemos reconocimientos de aprendizaje y demás. Y del otro lado, los pensamientos alternativos, las epistemologías no hegemónicas que se han configurado en esto que ustedes y yo hemos aprendido a reconocer en tiempos recientes como el pensamiento no hegemónico, alternatividad, modernidad, giro decolonial.

Lo que resulta de interés allí, y esto es para propiciar el diálogo, es si en la brecha del plano A y el plano B se configura la universidad que no está herida de muerte. La universidad en Occidente está ocupando lugares, en el caso particular de América Latina el lugar de la institucionalización; y entonces, de lado y lado observamos elementos para la conversación, por ejemplo, del plano A, recientemente se ha publicado un informe en el que indica hacia dónde debe ir América Latina, y puso cinco retos a la tradición del conocimiento en América Latina, dijo que las tecnologías, que el consumo de drogas, que el ascenso del narcotráfico a la política, que la internacionalización y que el respeto por la globalización son cinco elementos que América Latina ha de reconocer como formas de reconocimiento o formas de integración de la universidad mediante la sociedad.

Pero también se ha dicho que los países latinoamericanos deben hacer la tarea juiciosa de acercase a los modelos occidentales de desarrollo. Así mismo, cada una de estas estrategias nos está indicando que el plano A tiene una hegemonía y que la universidad es su extensión, y que entonces en el detalle las universidades de este tiempo en América Latina están diciendo que cumplen con modelos, créditos, protocolos, con Colciencias y con las reglas del juego, y entonces allí hay una universidad que está indicándonos rutas, horizontes y mensajes a institucionalizar la forma de ver el mundo.

Lo que resulta de interés en ese plano A es observar que la universidad se ha despojado de Occidente y ella misma es una institución que contiene lenguajes y nomenclaturas propios de disposición y de control, y el objetivo de este panel es, ¿cuáles son los desafíos de la universidad hoy?

En el plano B, entonces, Ramón Grosfoguel ha indicado que Inglaterra, Italia, Francia, Alemania son Occidente y que en esa medida será muy importante propiciar pensamientos alternativos, epistemologías no hegemónicas, y pensar entonces que la universidad latinoamericana es alternativa. En esta mirada, la pregunta es ¿qué hay en la brecha de estos planos A y B para la universidad? Y aún, hoy muchos han planteado que las 
experiencias y el contexto determinan la nueva cosmovisión de la universidad. En sus planteamientos indicaran que la universidad es responsable de la construcción de lo público, entonces, en el marco de ese plano A y ese plano B, de esa brecha, ¿cuál es la responsabilidad de la universidad en América Latina para construir lo público?

\section{Contextualización acerca de la pregunta}

Se sabe que la universidad está dentro de la sociedad, y entonces la universidad es como un espejo, a modo de fragmentos, que también refleja la sociedad en la que participa, en esa medida no sería tan taxativa en la interpretación que compromete la responsabilidad en la construcción de lo público sólo a la universidad, sino que a propósito del panel hacemos un recorte favorecido por la provocación de la invitación en el tema que tenemos, y con seguridad también, la participación en un debate que tiene un foco próximo desde el lugar que ocupamos en nuestro desempeño desde universidades. Cuando la maestra Marta Cardona manifestó "vamos a invitar a gente que está en las universidades, que participa en redes de cooperación", es decir, hubo ya una intencionalidad también en la invitación.

La cuestión destaque, en relación a la pregunta es el consenso de que el ser humano está atravesado por ambigüedades, es decir, si pensamos la universidad, la universidad con el cuerpo palpitante de los sujetos que la construimos, pues entonces también estamos marcados por la ambigüedad, estamos marcados por la necesidad de trascender, pero también estamos marcados por la contingencia que nos determina en la construcción histórica, ¿por qué eso?, porque cuando participamos de la construcción de un grupo de investigación y del metabolismo en el que estudiantes y profesores, así como pares, desde la región, el país, la red internacional, intentamos situarnos en los debates para problematizar de modo diverso el complejo mundo en que nos ha tocado vivir.

Hemos estado discutiendo, en el evento "Epistemologías del Sur para germinar" en la Universidad Pontificia Bolivariana-UPB (2014) temas que tenían que ver con la teoría de la complejidad, y fue muy interesante el debate, porque hubo un momento en el que se afirmó: la ciencia parte en la década del cuarenta del siglo XX con la ciencia de la complejidad, antes de eso lo que hay es un equívoco en lo que hemos considerado como ciencia (GIT, 2014). Entonces hubo un debate, alguien que estaba entre el público pregunta “¿y cómo pensamos las comunidades tradicionales, ¿cómo pensamos los campesinos, ¿cómo pensamos la población que vive en condiciones de sobrevivencia?”. Empezaron a surgir diversas intervenciones y eso se volvió una tensión muy interesante, porque piensen que desde aquí hay por lo menos una 
preocupación por el enfoque desde uno y otro lado, pero allí hubo un debate profundo donde también se cuestionaba hasta dónde y desde dónde se pueden plantear teorías únicas, para interpretar el mundo.

Cuando se habla de alternativo o cuando se habla de la relación con la praxis, eso también se puede volver una confusión, es decir, el planteamiento atravesado por la delicadeza de la oportunidad de poner una provocación en la mesa, pero sabemos que somos seres profundamente contradictorios, lo que también nos enriquece.

Es muy riesgoso decir que la proximidad con la praxis es per se la garantía de acertar en la construcción que hacemos desde la universidad, como es riesgoso también pensar que solamente construir la teoría ya no desde el pensamiento occidental nos va a hacer más potentes para interpretar la realidad, la cosa es mucho más compleja; diríamos, y ahí recorremos a Santos (2011) por su posición como sujeto histórico en una coyuntura especialmente difícil del Brasil y su concepción del espacio y del lugar, para hablar desde la necesidad de trabajar en clave del territorio y desde los lugares donde se vive el cotidiano. Entonces, es decir que en toda esta búsqueda que estamos teniendo, más allá de buscar la estrella que nos ilumine, está la capacidad de la empatía, la capacidad de reinterpretar, y diríamos, de recrear desde la coyuntura de la realidad de nuestro tiempo, porque claro, participamos, estamos de paso, pero es un tiempo histórico.

Cuando la teoría se reproduce a través de modas que parecen imponer la condensación de realidades, hay que sospechar de esos contenidos y de la intencionalidad que vinculan. Por posmodernidad se entiende una corriente de pensamiento que está saturando las ciencias sociales y que viene cuestionando el proyecto científico heredado de la Ilustración y constitutivo de la modernidad. Se propone rechazar verdades universales. La ciencia sería un juego de lenguaje, constituido por saber y poder, privilegiando formas de conocimiento diverso. Cuestionando de ese modo los grandes relatos y negando la perspectiva ontológica de la procesualidad histórica para entender el mundo contemporáneo. Y la ciencia se reinterpretaría negando los presupuestos del mundo occidental, discursos que son producto de la intencionalidad colonizadora. Pensar desde las clases subalternas y comunidades minoritarias seria plantarse desde los poderes verídicos y legítimos. Sería la postura decolonial de la ciencia. Este se instaura como un "deber ser". Mientras estas ofensivas se masifican en los discursos académicos, la materialización de la vida continua, con una profunda desregularización de la economía, de la política, de la educación, de los derechos, de cara a la fragmentación de la vida social. 
Lo que se piensa es que los lugares, lo palpitante de lo que está pasando en ellos, las itinerancias, las compenetraciones, este concepto que Gramsci denominaba los intelectuales orgánicos para referirse al vínculo entre quienes nos movemos por ejemplo en el medio de la academia y la vida de las poblaciones, con sus reivindicaciones y luchas, de esa empatía, deberán construirse caminos compartidos y búsquedas por sentidos de existencia humanizados. No hay ninguna ilusión en que sea desde los personajes más citados del mundo académico donde se van a recrear las contra-corrientes frente a diversos modos de barbarie. El revolcón va a venir desde abajo y por eso traigo a Santos (2011), cuando él piensa otros mundos posibles él dice que va a ser exactamente en los escenarios del conflicto de las reivindicaciones, de lo que hemos intentado banalizar, que es exactamente los escenarios de dolor, de tensión, de conflictos.

La discusión de Occidente no es tan sencilla como para plantear "es que las universidades simplemente son occidentales" y ese tipo de discusiones no cuadra, porque no se corresponden con la realidad. En realidad, si el mundo está complejo, si el mundo necesita respuestas complejas, las universidades no pueden ser simples, tienen que ser igualmente complejas, y ser complejas quiere decir que no debe haber discurso hegemónico en ninguna universidad, ni pública ni privada, el primer criterio para ser serios con la realidad que tenemos es la pluralidad, la diversidad.

Una de las grandes estrategias del mundo globalizado de hoy consiste en colonizar uno de los últimos territorios que necesitan para el diseño de mundo que tienen en la cabeza, y ese es el territorio del conocimiento, el cual en buena medida pasa por el mundo de las universidades.

Si algo se está "cocinando" es precisamente porque la universidad no solamente fue un espacio reproductor de Occidente, fue un espacio complejo, contradictorio, por eso es que el pensamiento crítico está presente en los ámbitos del conocimiento a lo largo de la historia universitaria, no ahora, no es que el pensamiento crítico se va a formar ahora, si fuera así la tendríamos muy difícil.

Ahora, el pensamiento crítico es paralelo, se ha dado sistemáticamente al lado de la consolidación institucional y autoritaria de los saberes, por eso es que es posible que las universidades sean espacios de vida, de contradicción, de debate, y por eso es que ahora se le pretende convertir al extremo en norma, institucionalidad, cuantificación, indicadores de productivismo.

Por eso, no hay la ilusión en que sea la universidad el escenario o medio desde donde se construya un contra-movimiento a todo esto que reivindica pensamiento único. La 
universidad se debe alargar en sentidos, ampliándose en la sociedad de su tiempo, Mançano (2015).

Estas reflexiones, son también la convicción de que la universidad en el 2030 existirá, sin embargo su misión/alcance de inspirar sueños estará en franca dependencia con la pertinencia para compenetrarse con los dilemas futuro presente. Naturaleza cosmopolita en la concepción del mundo, de su diversidad, de la necesidad inaplazable que tiene la universidad de producir teoría, en arduo trabajo basado en cooperaciones multidisciplinarias, de cara a redescubrir la lucidez, el coraje y la esperanza que requiere el mundo presente, enfrentando las aversiones contemporáneas por todo lo que se interpreta como objetividad, sintetizado en los lemas de la autonomía de las representaciones, cuando un eco repite como moda que no existen hechos, solo existen interpretaciones y por ende la incredulidad en la potencialidad del saber científico, con toda la fuerza del antihumanismo basado en la muerte del sujeto. ¿En qué momento histórico abandonamos el humanismo y nos quedamos con sus fetiches? ¿Por qué negamos la centralidad del proceso de individuación social?, ¿por qué tememos instaurar revoluciones sociales como necesidad permanente e infinita de recrear la vida?

\section{La universidad intercultural desescolarizada}

Para lograr los objetivos de una educación más humanista y pluralista, menos escolarizada y/o estructuralista se hace necesario, según Iván Illich (1985, p. 145):

[..] que se apliquen unas garantías constitucionales. A saber: los alumnos no podrían ser sometidos a un programa obligatorio, a una discriminación por no poseer un certificado o diploma; además, no habría que mantener planteles educativos, ni educadores, sino que usarían la tecnología moderna para lograr que la libre expresión, la libre reunión y la prensa libre fuesen realmente universales, $y$, por consiguiente, plenamente educativos.

El autor hace referencia a las posibles alternativas para una educación humanizada, que desarrolle el sujeto en su totalidad, en la medida en que el alumno/estudiante, se torna objetivamente el sujeto del proceso educativo, es decir, las mediaciones son a partir de y para él, y no umbilicalmente ligadas a la lógica institucional vigente. Dialécticamente, es también una crítica al modelo de concepción educativo basado en instituciones estructuralistas cuya premisa es que las personas tengan que obligatoriamente estar - desde niños hasta edad adulta - en escuelas, siendo ensañadas, encuadradas en las reglas de las escuelas, que todavía en la contemporaneidad son reguladas por una lógica, en la cual el resultado de la asistencia es un aprendizaje valioso, el valor del sujeto aumenta con la cantidad de información concedida y puede medirse y documentarse mediante diplomas (BERNHEIM; CHAUÍ, 2008). 
En ese sentido, romper con los imperativos valorativos contemporáneos a través de la educación es también romper con los actuales medios de aprendizaje. Es decir, la escuela dejaría de ser "el proceso que especifica edad y se relaciona con maestros y exige asistencia a tiempo completo a un currículo obligatorio" (MEJIA; SALAZAR, 1986, p. 143) y pasaría a ser más una alternativa de aprendizaje en que los maestros estuviesen en la condición de "coadyuvantes" (así deben ser) tornándose más un sujeto estimulador del conocimiento y no de imposición o centralidad. Como sostiene Paulo Freire "a educação constitui-se em um ato coletivo, solidário, uma troca de experiências, em que cada envolvido discute suas ideias e concepções..." y además [...] "O que importa é que os professores e os alunos se assumam epistemologicamente curiosos" (FREIRE, 1998, p. 96). Finalmente facilitarían al estudiante, a través de los intercambios, conocer otros lugares, otras culturas permitiendo su desarrollo personal y colectivo. Como la llama Illich (1985), esta sería la "nueva escuela", pedagógicamente actuando para el proceso de transformación social.

Sin embargo, el conocimiento es más que instrucciones, hay que revivir el sujeto. La humanidad necesita de sujetos pensantes en una perspectiva integradora, más humanista y menos estructuralista, este es uno de los desafíos impuestos a la actual sociedad.

La experiencia laboral/académica de más de diez años entre Universidades latinoamericanas, los trabajos de cooperación internacional con varios países de la América del Sur y europeos evidencian la importancia de la internacionalización de la enseñanza, son ejemplos que reafirman la misión de los mestres: fundamentalmente formar nuevas personas con la idea de la interculturalidad (GOMES, 2016). Específicamente en relación con Suramérica, el convenio entre Brasil y Colombia, los resultados para los estudiantes y profesores hasta el momento, en términos de aprendizaje son significativos, y sobretodo hay un retorno para la sociedad de las investigaciones, por tanto, una forma de interacción social entre la academia y los ciudadanos. Sin embargo hay que considerar que es preciso que las academias, en la condición de espacios posibles para el desarrollo de estas experiencias, asuman sus responsabilidades en cuanto instituciones sociales y hagan esfuerzos cada vez mayores, pues "nosotros latino americanos, no conocemos nuestras realidades" suficientemente (https://www.youtube.com/watch?v=npXvvE8_gOc\&t=17s).

Stallivieri (2014) en su ensayo O Processo de Internacionalização nas Instituições de Ensino Superior sostiene que el proceso de cooperación internacional es esencial para la democratización del conocimiento. Hace sugerencia a la importancia histórica de las llamadas "universitas", que en la edad media concede la universalidad como una multiplicidad de visiones del mundo, comprensiones filosóficas, tendencias científicas y políticas, en resumen, 
diferentes perspectivas de pensar de los seres humanos, oriundos de varias partes del mundo. En la concepción de la autora, las universitas crearon en su contexto histórico condiciones humanistas de desarrollo posibilitando mayor calidad de vida para las poblaciones, y en la actualidad reflexiona sobre una cuestión fundamental: "Faz-se necessário repensar o papel e a importância das universidades, imbuídas de sua função integradora de culturas e de pessoas, - questão que, no mínimo, apresenta-se como provocadora” (STALLIVIERI, 2014, p. 2).

De esta forma, a través de la interacción e intercambios culturales entre los que hacen y comparten la educación ("mestres, alumnos y sociedad”), el conocimiento queda más libre, más humanizado y, por consiguiente, menos manipulado. Por tanto, retirando de él, el concepto institucionalizado, dentro de los muros de la escuela, como si fuera encerramientos residenciales (MEDEIROS et al., 2008) individualizados por sus propias ideologías y alienaciones. No obstante, como susteine Illich $(1985$, p. 70$)$ para dar este salto, es necesario que tengamos consciencia de que:

Cada uno de nosotros es responsable de su propia desescolarización, y sólo nosotros tenemos el poder de hacerlo. No puede excusarse a nadie si no logra liberarse de la escolarización. El pueblo no pudo liberarse de la Corona sino hasta que al menos algunos de ellos se hubieron liberado de la Iglesia establecida. No pueden liberarse del consumo progresivo hasta que no se liberen de la escuela obligatoria.

Para lograr tal desiderátum la técnica estaría vinculada al uso de las tecnologías, la cual "sirve para simplificar la sociedad y hacerla más transparente, de tal manera que todos los hombres puedan conocer de nuevo los hechos y las herramientas que modelan sus vidas" (MEJIA; SALAZAR, 1986, p. 146). De esta manera, sería posible concebir el conocimiento a través de la educación dentro y fuera de las instituciones, fortaleciendo enlaces que contribuyan para la formación plena del individuo y de la sociedad.

\section{Consideraciones finales}

La universidad en la condición de institución social y destinada a procesos de educación democrática necesita abandonar ciertos conceptos intencionalmente construidos a lo largo de la historia y acercarse con urgencia de la sociedad. Su desafío está en la necesidad de romper con los paradigmas del conocimiento estructuralista, que en la contemporaneidad homogeniza la forma como se construyen individuos y sociedad.

Las llamadas innovaciones tecnológicas por su vez, así como la noción de sociedad del conocimiento precisan ser revistas con urgencia. Las universidades caminan a pasos apresurados, tendencia y linealmente - por medio de la unión de los anteriores - para el 
productivismo, que sin embargo están produciendo abstracciones, contenidos mediáticos y superficiales que en grande medida no logran transformaciones sociales.

Hay una necesidad urgente e inconteste de romper con la lógica actual aparentemente irreversible - de educación ubicada al capital. El desafío, segundo Mészáros (2008), está mediante a la creación de una alternativa educacional distinta, en el sentido emancipatorio, cuja totalidad de las practicas educacionales sean capaces de reivindicar "uma educação plena para toda a vida, para que seja possível colocar em perspectiva a sua parte formal, a fim de instituir, também aí, uma reforma radical” (MÉSZÁROS, 2008, p. 55), teniendo en cuenta que la educación es un camino, por donde estratégicamente, es posible cambiar objetivamente las condiciones actuales de reproducción, en la medida en que los individuos, envueltos en la lucha por la construcción de una nueva orden social metabólica radicalmente diferente, también se auto cambien.

Finalmente, el dilema histórico de la sociedad definido por la crisis estructural del capital global, periodo donde se evidencia una condición histórica de transición, se evidencia también un espacio histórico y social abierto a la ruptura con la lógica del capital y en la elaboración de planes estratégicos ubicados a una educación para más allá del capital. En ese contexto, la tarea educacional se convierte en una incumbencia de transformación social, amplia y emancipadora, por tanto, los desafíos de las universidades Latinoamericanas están vinculados a la compresión de que la educación tiene que ser articulada y redefinida en su interrelacionamiento con las condiciones cambiantes y con las necesidades de la transformación social emancipadora e progresiva en curso.

\section{Referências}

\section{BERNHEIM, C. T.; CHAUÍ, M. S. Desafios da universidade na sociedade do}

conhecimento: cinco anos depois da conferência mundial sobre educação superior. Brasília: UNESCO, 2008. Disponíble en:

<http://unesdoc.unesco.org/images/0013/001344/134422por.pdf〉. Acceso jul. 2017.

FATTORELLI, Maria Lúcia. Crise, escândalos midiaticos e financiamento de campanha engordam o sistema da divida às custas do povo. Entrevista. Revista Caros Amigos, São Paulo, p. 13-17, nov. 2015.

FREIRE, P. Política y educación. México: Siglo XXI, 1993.

FREIRE, Paulo. Pedagogia da autonomia. 9. ed. Rio de Janeiro: Paz e Terra, 1998. 
GOMES, Edvania T. A. Importancia de las cooperaciones internacionales para la educación superior. In: GRUPO de investigación sociedad y naturaleza Nexus. Diponible en: $\langle$ https://www.youtube.com/watch?v=npXvvE8_gOc\&t=17s $>$. Acceso en: 28 mayo. 2017.

GIT - Grupo de Investigación Territorio. Epistemologías del Sur para germinar. Universidad Pontificia Bolivariana, Campus de Laureles, Medellín, 2014.

ILLICH, Iván. La sociedad desescolarizada. México, 1985. Disponible en: <http://www.mundolibertario.org/archivos/documentos/IvnIllich_lasociedaddesescolarizada.p df>. Acceso en: 28 jun. 2017.

MANÇANO, F. B. Camponeses na universidade: a criação do Mestrado em Desenvolvimento Territorial na América Latina e Caribe. Carta Económica Regional, Jalisco, v. 27, n. 115, ene./jun. 2015. Disponible en:

<http://www.cartaeconomicaregional.cucea.udg.mx/index.php/CER/article/view/5669l>. Acceso en: 13 jun. 2017.

MEDEIROS, C. R. O.; et. al. Condomínios horizontais fechados: segregação do espaço social. Revista Eletrônica de Administração, Franca, v. 11, n. 12, jan./jul. 2008. Disponível em: <http://periodicos.unifacef.com.br/index.php/rea/article/viewFile/210/62>. Acceso en: 28 jun. 2017.

MARX, K. El capital. Chile: CEME, 1867. T. 1. El proceso de acumulación capitalista.

MEJIA, Gloria Maria; SALAZAR, Nohora Rico (Org.). Experiencias educativas del siglo XVIII y principios del siglo XIX. Bogotá: Pontificia Universidad Javeriana, 1986.

MÉSZÁROS, István. A educação para além do capital. São Paulo: Boi Tempo, 2008.

RAMONET, I. El Mundo en 2030. Le Monde Diplomatique, n. 211, mayo 2013. Disponible en:

<http://www.mondediplomatique.es/?url=editorial/0000856412872168186811102294251000/ editorial/?articulo=e8187d95-88cd-46a5-a22e-d69e48ef3c63>. Acceso en: 13 mayo. 2017.

SANTOS, M. Documental: O mundo global visto do lado de cá. Disponible en: <https://www.youtube.com/watch?v=-UUB5DW_mnM>. Acceso en: 15 mayo. 2017.

STALLIVIERI, Luciane. O processo de internacionalização nas instituições de ensino superior. Caxias do Sul, 2014. Disponible en:

<http://iglu.paginas.ufsc.br/files/2014/08/SLIDES-LUCIANE.pdf>. Acceso en: 28 mayo. 2017.

UNESCO. Situación Educativa de América Latina y el Caribe. Hacia una Educación para Todos 2015. Disponible en:

<http://www.unesco.org/new/fileadmin/MULTIMEDIA/FIELD/Santiago/pdf/situacioneducativa-mexico-2013.pdf> . Acceso en: 13 mayo. 2017.

WALLERSTEIN, I. Analisis de Sistemas-mundo. Siglo Veintiuno Editores, 2015.

Disponible en: <http://www.multiversidadreal.edu.mx/wpcontent/uploads/2015/09/Wallerstein-Immanuel.pdf>. Acceso en: 19 jul. 2017.

ZIBECHI, R. Otros mundos posibles. Revista Kavilando, Universidad San Buenaventura, Medellín, v. 1, n. 06, julio 2015. Disponible en: < http://revistakavilando.weebly.com/revvolumen-6-nuacutemero-1.html>. Acceso en: 25 mayo 2017. 
${ }^{1}$ Aura González Serna Universidad Pontificia Bolivariana | Faculdade de Trabajo Social| Escuela de Ciencias Sociales Medellín | Colômbia. Contato: aura.gonzalez@upb.edu.co ORCID (iD https://orcid.org/0000-0002-3285-863X

${ }^{2}$ Edvânia Torres Aguiar Gomes Universidade Federal de Pernambuco | Ciências Geográficas Pernambuco |PE | Brasil. Contato: torres@ufpe.br ORCID ID https://orcid.org/0000-0002-0865-4805

${ }^{3}$ Dweison Nunes Souza Silva Universidade Federal de Pernambuco | Secretaria de Educação de Pernambuco | Biologia Pernambuco | PE | Brasil. Contato: dweison@gmail.com ORCID (iD) https://orcid.org/0000-0002-4720-4022

Artigo recebido em 6 de setembro de 2017 e aprovado em 21 de fevereiro de 2018. 